\title{
Characterization of N200 and P300: Selected Studies of the Event-Related Potential
}

\author{
Salil H. Patel ${ }^{1}$ and Pierre N. Azzam ${ }^{2}$ \\ 1. The Methodist Hospital, Houston, TX 77002, USA \\ 2. Baylor College of Medicine, Houston, TX 77030, USA
}

Corresponding address: Salil H. Patel, MD, salil.patel@christushealth.org / sp.jhem@gmail.com. Tel: 17137577529. Fax: 1713 6577208

Received: 2005.08.08; Accepted: 2005.09.15; Published: 2005.10.01

The Event-Related Potential (ERP) is a time-locked measure of electrical activity of the cerebral surface representing a distinct phase of cortical processing. Two components of the ERP which bear special importance to stimulus evaluation, selective attention, and conscious discrimination in humans are the P300 positivity and N200 negativity, appearing 300 $\mathrm{ms}$ and $200 \mathrm{~ms}$ post-stimulus, respectively. With the rapid proliferation of high-density EEG methods, and interdisciplinary interest in its application as a prognostic, diagnostic, and investigative tool, an understanding of the underpinnings of P300 and N200 physiology may support its application to both the basic neuroscience and clinical medical settings. The authors present a synthesis of current understanding of these two deflections in both normal and pathological states.

K eywords: Electroencephalography (EEG), N2, N euroimaging, P3, Selective attention

\section{Introduction}

The widespread adoption of electroencephalography (EEG) for the non-invasive assessment of cortical activity has inaugurated a distinct era in the elucidation of brain function. Due to its high temporal resolution, EEG imaging of relative scalp electrical positivities and negativities may expose subtle cognitive activity. Indeed, shortly after the advent of electrophysiological recording in the late 1920s, physiologists readily observed discrepancies in the brainwave records of normal subjects compared to those with documented illness [1]. Developments in both EEG acquisition technology and data processing capability have allowed for the identification and characterization of specific deflections comprising the activity associated with a given experimental stimulus or response. This specific subrecord of time-locked data is identified as the EventRelated Potential (ERP). Two constituents of the ERP, the N200 and P300, appear to be closely associated with the cognitive processes of perception and selective attention (Figure 1).

Through various experimental paradigms, topographic mapping of averaged signals (Figure 2), and electrical source analysis, it has been possible to assemble a sizable knowledge-base regarding these ERPs. Prior reviews of the basic physiology underlying these potentials [2, 3] have laid the groundwork for understanding the vast literature in the field; the objective of this article is to briefly introduce the characteristics of these ERP components, extending to current experimental findings, and to describe changes observed during pathophysiologic states. Such an approach is particularly relevant considering the growing interest in the application of ERP analysis for disease screening, risk stratification, and as indices of progression.

\section{The $\mathbf{N 2 0 0}$}

Typically evoked 180 to $325 \mathrm{~ms}$ following the presentation of a specific visual or auditory stimulus, the N200 (or N2) is a negativity resulting from a deviation in form or context of a prevailing stimulus [4]. Elicitation may be achieved through an experimental oddball paradigm, in which subjects are exposed to a continuous succession of two types of stimuli, one presented regularly and the other displayed sporadically (Figure 2). Upon the presentation of the rare stimulus following a string of standard stimuli, the N200 is observed [5]. A number of investigations have utilized a variation of this paradigm, an oddball detection task, in which the subject is asked to physically respond to the deviant stimulus. A number of studies cited in this review incorporate the oddball paradigm, in part due to its widespread prevalence, reproducibility, simplicity, and applicability across sensory modalities. In these experiments, the N200 is typically evoked before the motor response, suggesting its link to the cognitive processes of stimulus identification and distinction [4].

\subsection{N2 Sub-Components}

Several distinct N200 potentials have been characterized [5]: one set reflecting involuntary processing, another evoked through active processing. In repetitive stimulus-presentation, the $N 2 a$ is an anterior cortical distribution evoked by either conscious attention to, or ignoring of, a deviating stimulus [6]; the $N 2 b$ is a negativity of central cortical distribution seen only during conscious stimulus attention; the N2c arises frontally and centrally during classification tasks [3]. Furthermore, stimuli presented in visual search tasks with specific laterality and which are task-relevant may evoke an $N 2 p c$ deflection, as an index of attentional shift, in the occipitaltemporal region of the contralateral cortex [7].

\subsection{MMN and stimulus variation types}

The mismatch negativity (MMN), or auditory $\mathrm{N} 2 \mathrm{a}$, is elicited in a task-independent manner by auditory oddball detection paradigms and is believed to reflect disparity between the deviating stimulus and a sensory-memory representation of the standard stimulus [8]. Thus, MMN data provide a means by which to analyze the characterization of auditory stimulus features in sensory 
memory [9]. Picton et al., in an excellent review, note that since the MMN is generated regardless of attention to stimuli, it likely represents an automatic novelty-sensing process [10].

Additionally, a posterior negativity has been observed in response to alteration of visual stimuli, and has been proposed to be a visual counterpart to the auditory MMN, dubbed vMMN and appearing approximately 120-200 msec post-stimulus [11]. The existence of such an ERP is supported by other experimental observations [12], with the prestriate region is the likely source, as localized by color discrimination tasks [13].

Combined brain-lesion studies and functional mapping have established the primary role of the auditory temporal cortex in MMN generation, supporting the independent storage and examination processes of auditory stimuli in the auditory cortical region [14]. Evidence also suggests frontal-lobe involvement in MMN generation, perhaps the involuntary switching of attention due to a stimulus change, with thalamic and hippocampal generation of possible MMN subcomponents [8].

The effects of variant auditory stimulus conditions as intensity, presentation rates, and location on the MMN component have been studied extensively. The MMN has been elicited under the oddball paradigm through both increases and decreases in stimulus intensity [15]. In addition, MMN latencies have been found to increase with increased standard-deviant intensity deflections, reflecting an elevated cognitive processing requirement for more extensive stimulus deviations [16].

MMN data has also been utilized to characterize auditory processing duration. In one particular double deviation paradigm, a string of standard stimuli composed of two constituents, an introductory tone of invariable frequency and a subsequent frequency glide, were sporadically interspersed by a stimulus which deviated from the standard in the intensity of the first component and the glide direction of the second constituent. The number of distinct MMN components elicited by this double deviation was found to be dependent upon the presentation-duration of the initial stimulus constituent. Specifically, presentation times of less than $150 \mathrm{~ms}$ elicited a single $\mathrm{MMN}$, while presentation times of greater than $250 \mathrm{~ms}$ evoked two distinct MMN peaks, providing significant evidence for the processing of auditory information over 200-ms time frames [17].

A comparison of the effects of different auditory stimulus deviation types on the MMN component has been determined through a number of investigations utilizing the auditory oddball paradigm. In one such study examining frequency and intensity deviation, changes in inter-stimulus interval (ISI) and intensity in auditory oddball detection tasks evoked two different MMNs based on whether the deviant stimulus was defined by frequency or by intensity. While the MMN elicited from the presentation of the infrequent stimulus was not affected by intensity and ISI variation, both stimulus conditions significantly altered the MMN component evoked in the intensity-divergence condition, providing evidence for central differences in frequencyevoked and intensity-evoked MMN [18].

\subsection{MMN, age, and cognition}

The effects of aging on analytical cognitive operations have become a prominent focus of research. Aging studies have shown that, using high (i.e. 3 to 8 second) inter-stimulus intervals (ISIs), MMN peak-areas decrease in the older population, suggesting a shortening of the sensory auditory memory trace with increasing age [19]. The same phenomenon occurs in the very young, indicating the necessity of maturational changes for the complete efficiency of the auditory sensory memory. In a similar oddball paradigm using short inter-stimulus intervals, however, MMN latency and amplitude varied little as a result of increasing age, suggesting the invariance of automatic stimulus analysis and auditorymemory-based comparison in this condition throughout the lifetime [20]. In similar studies on musical subjects using high ISIs, a clear link between musicality and larger MMN amplitudes suggests that musical subjects possess enhanced auditory sensory memories as compared to nonmusical individuals [21].

$2.4 N 2 b$

A second N200 sub-component, the N2b, corresponds to voluntary processing and is elicited when subjects selectively attend to deviations in oddball paradigms. Unlike the $\mathrm{MMN}$, the $\mathrm{N} 2 \mathrm{~b}$ is not restricted to auditory tasks and does not specifically reflect departure from a collection of standard stimuli. Rather, the N2b is elicited by template mismatch, or deviation from a mentally-stored expectation of the standard stimulus [16]. Investigations in $\mathrm{N} 2 \mathrm{~b}$ scalp distribution have suggested the centrality of the frontal and superior temporal cortex for generation [22]. In addition, by association with color selection, the $\mathrm{N} 2 \mathrm{~b}$ has also become affiliated with general detection processes controlled at the level of the anterior cingulate cortex [23]. The $\mathrm{N} 2 \mathrm{~b}$ is associated with an inferior anterior ERP positivity, the P2a [24]. This relation is postulated to represent the interaction between areas of salience representation and feature representation in the cortex [25].

\subsection{N2b and stimulus variation types}

Despite a relatively recent growth of interest in $\mathrm{N} 2 \mathrm{~b}$ characterization, a number of studies have probed the effect of stimulus and experimental condition variations on this N200 sub-component. Unlike the MMN, the N2b has been found to reflect alterations in orthography, phonology, and semantics in addition to visual and auditory deviations [26]. N2b amplitudes have been studied extensively, with greater standard-target variation being linked to increases in $\mathrm{N} 2 \mathrm{~b}$ amplitude. This phenomenon demonstrates, as with the MMN, the increase of cognitive processing requirements with greater stimulus-deviant deflections. In oddball detection tasks, in which the subject actively attends to the presented stimuli and responds only to the deviant ones, elicitation of the $\mathrm{N} 2 \mathrm{~b}$ has been proven possible at lower levels (i.e., at lower amplitudes) with missed targets and standard stimulus presentations. This suggests the contingency of the $\mathrm{N} 2 \mathrm{~b}$ amplitude not upon the recognition of the actual deviant targets but instead upon the plausibility of target differentiation [27]. In visual discrimination tasks, N2b amplitude is directly correlated with discrimination difficulty [28].

$\mathrm{N} 2 \mathrm{~b}$ and MMN data have been examined cooperatively in order to compare the possible constraints on active and automatic variation detection processes. 
Investigations have been conducted to determine the effects of ISI deviations on the amplitudes of MMN and $\mathrm{N} 2 \mathrm{~b}$ components evoked from a passive oddball paradigm and an active oddball detection task, respectively. ISI variations were found to have more notable effects on $\mathrm{N} 2 \mathrm{~b}$ amplitudes than corresponding MMN amplitudes, demonstrating the increased potential for conditions and limitations on controlled stimulusvariation identification processes as opposed to automatic, passive processes [18].

\section{$2.6 N 2 b$ and age}

A number of studies have investigated and proposed the effects of aging on the N2b component and, thus, upon selective information processing as a whole. In one oddball detection study involving the effects of color deviation on $\mathrm{N} 2 \mathrm{~b}$ elicitation in subjects from age 7 to age 24, increasing age was found to correspond directly to decreases in $\mathrm{N} 2 \mathrm{~b}$ latency and alterations to the component's physiological generation. This suggests the optimization of visual and cognitive discrimination processes as a result of physical maturation [29]. Furthermore, in an auditory oddball detection task in which the stimulus was characterized by two distinct features, N2b latency was found to increase significantly in the elderly. As the $\mathrm{N} 2 \mathrm{~b}$ reflects processing in attention, this suggests the general decay of attentional processes with age [30]. These results were taken further in another aging study comparing the MMN and N2b components elicited through tone deviations in a dichotic listening task on subjects from age 23 to age 77 . Specifically, while age had little, if any, effect on the latency and amplitude of the evoked MMN, the elicited N2b was found to continuously increase in latency and decrease in amplitude with increasing age. These findings suggest the constancy of automatic distinction and analytical processes over the lifetime and the weakening of controlled processes requiring selective attention with increasing age [20]. A similar study by Pekkonen, et al. resulted in the same conclusions; it was resolved that the increase in N2b latency and decrease in $\mathrm{N} 2 \mathrm{~b}$ amplitude reflects either a more rapid disintegration of the sensory memory expectation central to $\mathrm{N} 2 \mathrm{~b}$ generation or the decrease in processing acuteness with age [19].

\subsection{N2 Posterior ERPS}

In tasks of visual perception which involve the discrimination of a target presented in a field of a limited number of surrounding objects (i.e., a pop-out paradigm), a target with unique features is detected rapidly [31]. In a pop-out paradigm, form, color, and word discrimination tasks elicit the N2pc negativity [32]. This ERP disappears if the number of surrounding distractors increases above 120; however, a distinct posterior N2 distribution, the $\mathrm{N} 2 \mathrm{p}$, shows enhanced negativity with increasing set size and likely represents texture segmentation activity [33].

\section{The P300}

The classical P300 deflection emerges in a timelocked record as a positivity typically appearing approximately 300 to $400 \mathrm{~ms}$ following stimulus presentation. Timing of this component may range widely, however, from $250 \mathrm{~ms}$ and extending to $900 \mathrm{~ms}$, with amplitude varying from a minimum of $5 \mu \mathrm{V}$ to a usual limit of $20 \mu \mathrm{V}$ for auditory and visual evoked potentials, although amplitudes of up to $40 \mu \mathrm{V}$ have also been documented [34]. The P300, first described by
Sutton, et al. [35], is perhaps the most-studied ERP component in investigations of selective attention and information processing, due partly to its relatively large amplitude and facile elicitation in experimental contexts.

Most well-characterized is the P3b, or "classical P3" (N.B. the term P300 used subsequently in this review generally refers to this $\mathrm{P} 3 \mathrm{~b}$ sub-component), in contradistinction to the $P 3 a$, typified by shorter latencies and frontally-oriented topography [36, 37]. One possible interpretation of the P300 is that it reflects broad recognition and memory-updating processes, with the P3b proposed to reflect match/mismatch with a consciouslymaintained working memory trace, while the P3a reflects a passive comparator [6]. The frontal P3a may be elicited by the more infrequently-appearing stimulus of with a two-stimulus oddball task, regardless of attentional (i.e., target or nontarget) status [38]. The P3a has also been demonstrated experimentally in target/nontarget tasks modified to include an additional infrequent stimulus; confusion has arisen over the distinction of a separate anterior Novelty P3 observed in response to rare, completely unexpected stimulus in a modified oddball task (Figure 2) [39]. While ERP waveform factor analysis in dictates that the Novelty P3 and P3a are in fact identical [40], the application of cortical potential imaging methods to model responses to auditory stimuli supports the hypothesis of temporal- and spatial distinction of the Novelty P3 and parietal P300 [41]. Principal component analysis isolates the Supplementary Motor Cortex (SMC) or cingulate gyrus as generators for the Novelty P3 [42].

The P3b component has been proposed to index memory storage as well as serving as a link between stimulus characteristics and attention [6]. Markedly increased $\mathrm{P} 3 \mathrm{~b}$ amplitude is observed in response to the rare stimulus of an oddball experimental paradigm. Investigational evidence points to the explanation that P300 properties are affected by the nature of the stimulus [43]: factors documented to alter P300 amplitude include presentation probability [44], stimulus sequence (45), stimulus quality, attention, and task relevance of the stimulus [34]. Croft and colleagues contend that P300 amplitude is affected by the target-to-target time interval (TTI), and not independently by inter-stimulus time interval or stimulus probability [46].

Quadruple-dipole modelling of somatosensoryevoked P3b has localized its origin specifically to the hippocampal and parietal cortical regions [47]; a separate analysis of auditory-evoked potentials via brain electric source analysis and multiple-dipole modelling indicates putative generators in the hippocampus and temporal lobe [48]. Physical lesion corroborates these findings, with damage to tissue in the temporal-parietal junction inducing a loss of the P3b waveform $[49,50]$.

Invasive cerebral electrode recordings localize the temporal-parietal junction as the generator for the classical P300 [51]. Hoffman [4] proposed that P300 latency varies as a function of factors governing stimulus evaluation time. For example, the concomitant presentation of relevant stimuli and non-germane "distractor images" may notably increase latencies [52], although latencies have not been found to be altered in studies in which the relevance between stimulus and response is modified. In word vs. color "Stroop" tasks requiring a verbal response, slower reaction times are observed in response to non-matching word/color combinations, though these combinations yield no 
corresponding changes in latency [53]. More recent visitation of the Stroop paradigm, employing random stimulus-response mapping to buttons, has rendered similar results [54]. These data imply that the P300 is most likely not involved in response selection processes, but rather more upstream operations. P3 latency is indirectly related to TTI stimuli, regardless of modality $[55,56]$; these findings further support the concept of the P3 as a proxy for some element of stimulus evaluation time.

Evidence has accumulated describing the P300 as a cognitive routine supporting the formulation of an internal environmental model in which a stimulus be evaluated: i.e., the "context-updating" hypothesis [57]. This concept is reinforced by the direct relationship between P300 latency and subject reaction time [58]. Oliver-Rodriguez and colleagues suggest, from visual observation studies using cues of human faces, that the P300 is involved in stimulus evaluation to the extent that it triggers context-based updating [59]. Alternatively, the Context-Closure Theory [60] emerged as an alternative to context-updating theory; reflecting the concept that the P300 reflects activity of memory trace remodelling postdetection of a target stimulus [61].

It should be noted that ERP latencies fall into two distinct categories: active or passive, depending on the experimental paradigm. A passive latency may be elicited by presenting rare stimuli in oddball tasks without giving the subject instructions, while active latency is observed in tasks which require the subject to respond to the rare stimulus with button-presses.

In visual oddball tasks, observed active and passive latencies for the P300 are comparable, suggesting that use of visual-cue experimentation may be useful even for subjects who lack the motor capacity for stimulus response [62]. Observation of the P300 latency via auditory passive single-tone and passive oddball paradigms may similarly be effective when active responses are infeasible [63].

Further, P300 parameters may be affected by familiarization with repeated stimuli. Ravden and Polich [64] have demonstrated that, in oddball visual tasks requiring a motor response to targets, with targets representing $50 \%$ of all stimuli, the observed P300 amplitude decreases with repeated presentation of the target stimulus. It is significant that in this model similar habituation is was not identified for latencies of the P300 ERPs. Moreover, utilizing a paradigm in which targets are presented with only a 1 in 5 probability, habituation occurs only for standard stimuli, with target stimuli eliciting no decrease in P300 amplitude. At the same time, both target and standard stimuli elicit increasing latencies over large successive trial blocks [65].

How may these seemingly disparate habituation effects be reconciled? Since multiple-dipole modelling suggests that the source for both novel and repeatedstimulus P300s are the same [66], some unified process most likely controls elements of P300 activation.

From an attention-theory standpoint, one may envision a continuum of interconnected processing at the level of stimulus processing and response formulation. (N.B., this model is speculative and intended for primarily illustrative purposes). Once a stimulus is identified as either a target or nontarget (or novel and unexpected), a response, such as updating an element of working memory, may be envisioned to be furnished as part of a specified "pipeline" for execution of current stimulus- response mapped tasks (SRMTs). As some task-switching time factor may reasonably be associated with each change in active stimulus response, it is perhaps the taskswitching duration which serves as the critical element in determining latency of the P300 signal produced by neuronal groups of the corresponding stimulus-response subunit.

To illustrate: when two stimuli are presented with equal frequency, then stimulus-response mapping can occur at relatively consistent rates. If, however, one stimulus is presented more frequently than another, the period associated with task-switching increases, resulting in increased latencies for response to the infrequent stimulus. Such a conclusion would be supported by reports from Duncan-Johnson and Donchin [44] indicating that increasing stimulus probability reduces stimulusevaluation and response-production periods.

Note that this model accounts for changes in amplitude as a result of feedback from currently-active SRMTs in a fashion similar to latency modulation, if we assert that the SRMT is the amplitude source for the ERP. Thus, an infrequent target, whose corresponding SRMT has not experienced positive feedback to any significant degree, would elicit a strong, uninhibited response.

\subsection{Morphological and Developmental Groundings}

Attempts have been made to draw correlations between P300 and callosal size. The majority of these experiments have compiled relations between ERP characteristics and age, gender, or handedness. For instance, studies have shown that in both auditory and visual stimulus discrimination tasks, measured amplitude is greater, with smaller corresponding latencies, in lefthanded subjects versus right-handers. Similar differences in $\mathrm{P} 3 \mathrm{~b}$ characteristics have been noted between the two genders [67]. Any correlations between these data and corpus callosum mass is subject to contention, however. MRI imaging and analysis by Hopper et al. [68] showed no significant connection between gender or handedness and callosal size; the information available did demonstrate, however, an inverse relation between age and callosal size. Conversely, a subsequent review of MRI and post-mortem reports indicated a callossal volume relationship to handedness and gender [69]; the clinical significance of these findings remains unclear.

Clear support exists for age-related modulation of the P300 deflection. In visual tasks, latencies increase with age, although the precise correlative nature of age and latency time is not certain [70]. Additionally, the presentation of higher-difficulty tasks elicits significantly slower reaction times in older subjects, independent of the manner in which task difficulty is increased. Changes in P300 latency, however, do not tend to such exhibit taskindependence and thus remain contingent upon the precise nature of difficulty variation [71]. Investigation of the auditory-evoked potential in subjects varying in age from 20 to 88 has uncovered a linear direct dependence between both active and passive P300 latency and age; in this same study, levels of active latency were associated with concentration ability, and passive latency with verbal proficiency and recall [72]. The decreases in P3 and novelty P3 with increasing age, and indeed a similar attenuation in the MMN, correspond clinically to changes in orienting behavior observed in the elderly [73].

A pathway for the physiological development of the P300 has yet to be made clear. Evidence exists of a 
possible nascent precursor which is reflective of workingvisual-memory operations. Infants presented with visual stimuli, in a passive paradigm, exhibit a slow positive wave, which increases in amplitude in response to novel stimuli [74]. Young children exhibit identifiable visual P300s characterized by large latencies (similar to those of elderly subjects) but do not demonstrate a significant P300 in the frontal region. As subjects age, they exhibit P300s which are shifted in amplitude more toward the frontal region [75]. Consequently, the P300 is likely comprised at the cellular level by a series of neuronal subnetworks which develop at differing rates.

Traumatic or other insult to the prefrontal cortex is reflected in diminished amplitude of the novelty P3 response to a novel stimulus [76]. This amplitude change further correlates with reduced attentional shift towards novel stimuli [77], possibly clinically manifesting as apathy.

\section{$3.2 P 300$ and the $N 2 b$}

Data indicate that the P300 is involved not only in processes of working memory, but may interact with the $\mathrm{N} 2 \mathrm{~b}$ in the control of motor response to external cues. Through the use of a "GO/NOGO" paradigm, requiring subjects to identify targets either with a motor response or a suppression of activity, it is possible to examine the processes associated with voluntary movement. In a visual GO/NOGO test, the ordinarily-detected posterior parietal P300 is absent during NOGO responses. It is also notable that the detection of a frontal N2 is associated only with instances of a NOGO response. Thus, the P300 appears to be inhibited by the appearance of the $\mathrm{N} 2 \mathrm{~b}$ in tasks of motor activity suppression [78]. The N2 waveforms elicited in a GO/NOGO paradigm presumably index response inhibition, with the anterior cingulate proposed as the likely generator [79].

\subsection{P300 and Broader Aspects of Environmental Interaction}

P300 amplitude may reflect filtering and constructive processes - $c f$. , a study of suggestible subjects inducted into hypnotic states: subjects were prescribed to experience positive (entity-fabricative) or negative (entityobliterative) hallucinations while participating in visual and auditory experimental studies. During the times at which subjects underwent negative hallucinations, greater P300 amplitudes were evident, whereas positive hallucination was associated with lower amplitudes [80]. Thus, conscious or subconscious perceptual modulation may be associated with some element of P300 activity.

Additionally, environmental triggers may allow for the broad-based alteration of P300 activation. P300 activity is modulated by the internal physiologic state of subjects, from natural circadian and ultradian rhythms to levels of fatigue or physical activity, as noted in a comprehensive review by Polich and Kok [81]. An experiment following respective subject groups across two of the three winter, spring, and summer seasons indicates that elicited P300 amplitude is inversely related to the amount of seasonal ambient sunlight, with women experiencing larger shifts than men [82]. This change in amplitude is suggestive of a direct (e.g., psychobiological) or indirect (e.g., societal) alteration of cognitive strategies or pathways in relation to seasonal variations, and this change is further filtered in relation to the sex of the subject.

The effect of cortical perfusion and metabolic activity on the P300 is still poorly understood. P300 amplitude is increased acutely by aerobic exercise [83]; however, evidence suggests that food intake does not specifically affect P300 parameters in relation to other ERPs [4, 84].

\subsection{P300 in Pathologic Conditions}

The P300 has been applied in a wide array of clinical research settings. The practical utility in therapeutic contexts for measuring and tracking of ERP findings in cases versus controls for most such studies is unclear, as most extant studies are descriptive and limited in power and generalizability. Still lacking are large-scale, blinded, randomized, prospective studies of ERP-guided therapy; however with decreasing costs of signal acquisition and processing, such research may soon be practical.

\subsubsection{Schizophrenia}

A disease of cognitive disturbance involving multiple symptom complexes and variable course and presentation, schizophrenia is nonetheless a clinicallydiagnosed disease. Beginning in the 1970s, attempts have been made to objectify various aspects of the disease and its pathogenesis via analysis of the P300 [85], particularly in auditory stimulus modalities, have been reviewed extensively elsewhere [86]. It has been proposed that observed ERP abnormalities may reflect the observed defects in mnemonic binding and account in part for symptoms of reality-distortion [87].

\subsubsection{Endocrine/Metabolic Systems}

Local perfusion and substrate status in the brain modulate P300 characteristics. For instance, the postexercise period after aerobic muscle activity is associated with increased P3 amplitude, with respect to auditory discrimination [83]. Additionally, glycemic status of affects ERP parameters, as evinced by increased P3 latency as serum glucose levels fall below $3 \mathrm{mmol} / \mathrm{L}$ [88]. Increasing the oxygen content of blood plasma via exogenous epoetin, to increase hematocrit in anemic patients, has been correlated with decreased latency and increased amplitude of the P3 [89].

\subsubsection{Addiction}

P300 characteristics have been noted to differ in subjects who are either at risk off, or engage in, addictive behavior. P3b amplitudes have been demonstrated to be attenuated in individuals considered at high-risk for alcoholism, due to familial history, when compared to a low-risk group [90]. Similarly, lower P3a amplitudes have been noted in at-risk subjects [91]. In response to abstinence from alcohol intake, the P3b component remains depressed in amplitude [92]. It has been proposed that P3a abnormality in high-risk groups may reflect an underlying state of CNS dis-inhibition involved in the pathophysiology of the condition [93]. Genetic linkage studies involving families with a history of alcoholism show involvement of chromosomes 2 and 6 and possibly chromosome 13, with genetic coding sequences containing genes involved in the construction of ionotropic glutamate receptors and the acetylcholine receptor [94]; more recent work also supports linkage to chromosome 5 and chromosome 4 loci [95].

\subsubsection{CNS Parenchymal Disease}

P300 characterization has shed light upon diseases linked etiologically to deep brain structures, including the basal ganglia, as well as clinically-evident dysfunction of the superficial cerebral cortex, associated in particular 
with spreading and advanced disease. For instance, anterior $\mathrm{P} 3 \mathrm{a}$ is attenuated in amplitude in patients with Parkinson's Disease [96], with concomitant P300b anomalies [97]. Furthermore, differences in NOGO-P3 (and NOGO-N2) waveforms indicate dysfunctional frontal-lobar inhibitory processing [98], and may be useful as objective measures of Parkinsonian progression or functional limitation. Marked reduction or absence of P3 distributions in visual search tasks is observed in patients with the choreiform movement disorder Huntingtons Disease, which classically demonstrates caudate nuclear atrophy, but also may manifest cortical symptoms [99].

Among the most prevalent chronic disorders of cognition in the elderly is Alzheimers Disease, typically affecting temporal and associative cortex regions; prolonged P300 latency and attenuated amplitude have been observed in such patients [100]. It has been proposed that P300 activity may serve as a useful marker of attention and as a screen for combination-drug therapy in investigations of anti-Alzheimer drugs [101].

P300 latency may also be applied clinically as a diagnostic tool and a prognostic marker for recovery after cortical insult. A small study of patients with ischemic stroke has shown that changes in P300 latency correlated with subclincal damage to the right parietal lobe. Furthermore, magnitude of alteration in P300 in the subacute phase of stroke correlated with functional recovery after several months time [102]. Acute cortical damage to auditory processing structures might be assessed objectively in a complementary manner, via studies of the MMN [103].

\section{Closing Remarks}

Each of the specific components of the Event-Related Potential discussed in this review plays specific and significant roles in cognitive processes. While much progress remains to be made in terms of precise characterization of ERP components, especially of early negativities, their psycho-biological prominence is clear. Selective attention, stimulus response, and perceptual filtering in auditory, visual, and somatosensory domains may fall under the integrated purview of the MMN, N2b, and P300 phenomena. Future applications of ERP analysis as a psychological diagnostic and feedback tool are growing more and more promising, and as we gain more insight into the nature of the ERP, the search for clinical integration will assuredly intensify.

\section{Conflict of Interests} exists.

The authors have declared that no conflict of interest

\section{References}

1. Sabatini RME. Mapping the brain. Brain and Mind Magazine 1997; 3.

2. Pritchard WS. Psychophysiology of P300. Psychological Bulletin 1981; 89:506-540.

3. Pritchard WS, Shappell SA, Brandt ME. Psychophysiology of N200/N400: A review and classification scheme. Advances in Psychophysiology 1991; 4:43-106.

4. Hoffman JE. Event-related potentials and automatic and controlled processes. In: Rohrbaugh JW, Parasuraman R, and Johnson R Jr, Eds. Event Related Brain Potentials. New York: Oxford University Press. 1990:145-157.

5. Näätänen R, Picton TW. N2 and automatic versus controlled processes. Electroencephalography and Clinical Neurophysiology $1986 ; 38: 169-186$.
6. Näätänen $R$. The role of attention in auditory information processing as revealed by event-related potentials and other brain measures of cognitive function. Behavioral and Brain Sciences 1990; 13:201-288.

7. Luck S, Girelli M, Mcdermott MT, et al. Bridging the gap between monkey neurophysiology and human perception: An ambiguity resolution theory of visual selective attention. Cognitive Psychology 1997; 33:64-87.

8. Alho K. Cerebral generators of mismatch negativity (MMN) and its magnetic counterpart (MMNm) elicited by sound changes. Ear and Hearing 1995; 16:38-51.

9. Woods D. The physiological basis of selective attention: implications of event related potential studies. In: Rohrbaugh JW, Parasuraman R, Johnson R Jr, Eds. Event Related Brain Potentials. New York: Oxford University Press, 1990:3-18.

10. Picton TW, Alain C, Otten L, et al. Mismatch negativity: different water in the same river. Audiology Neuro-Otology 2000; 5(3-4):111139.

11. Czigler I, Balazs L, Winkler I. Memory-based detection of taskirrelevant visual changes. Psychophysiology 2002; 39:869-873.

12. Pazo-Alvarez P, Cadaveira F, Amenedo E. MMN in the visual modality: a review. Biological Psychology 2003; 63:199-236.

13. Czigler I, Balazs L, Pato LG. Visual change detection: event-related potentials are dependent on stimulus location in humans. Neuroscience Letters 2004; 364:149-153.

14. [Internet] Näätänen R. Mismatch negativity. http://www.psych.helsinki.fi/cbru/mmn.html

15. Snyder E, Hillyard SA. Long-latency evoked potentials to irrelevant, deviant stimuli. Behavioral Biology 1976; 6:319-331.

16. Sams M, Alho K, Näätänen R. Sequential effects on the ERP in discriminating two stimuli. Biological Psychology 1983; 17:41-58.

17. Winkler I, Czigler I, Jaramillo M, et al. Temporal constraints of auditory event synthesis: evidence from ERPs. Neuroreport 1998; 16:495-499.

18. Schröger E. The influence of stimulus intensity and inter-stimulus interval on the detection of pitch and loudness changes. Electroencephalography and Clinical Neurophysiology 1996; 100:517-526

19. Pekkonen E, Rinne T, Reinikainen K, et al. Aging effects on auditory processing: an event-related potential study. Experimental Aging Research, 1986; 22:171-184.

20. Amenedo E, Diaz F. Automatic and effortful processes in auditory memory reflected by event-related potentials: Age related findings. Electroencephalography and Clinical Neurophysiology 1998; 108:361-369.

21. Tervaniemi M, Ilvonen T, Karma $\mathrm{K}$, et al. The musical brain: brain waves reveal the neurophysiological basis of musicality in human subjects. Neuroscience Letters 1997; 226:1-4.

22. Potts GF, Dien J, Hartry-Speiser AL, et al. Dense sensor array topography of the event-related potential to task-relevant auditory stimuli. Electroencephalography and Clinical Neurophysiology 1998; 106:444-456.

23. Lange JJ, Wijers AA, Mulder LJ, et al. Color selection and location selection in ERPs: Differences, similarities, and neural specificity. Biological Psychology 1998; 48:153-182.

24. Potts GF, Liotti M, Tucker DM, et al. Frontal and inferior temporal cortical activity in visual target detection: Evidence from high spatially sampled event-related potentials. Brain Topography 1996; 9:3-14.

25. Potts GF, Tucker DM. Frontal evaluation and posterior representation in target detection. Cognitive Brain Research 2001; 11:147-156.

26. Sanquist TF, Rohrbaugh J, Syndulko K, et al. An event-related potential analysis of coding processes in human memory. Progress in Brain Research 1980; 54:655-660.

27. Loveless LE. Potentials evoked by temporal deviance. Biological Psychology 1986; 22:149-167.

28. Senkowski D, Hermann CS. Effects of task difficulty on evoked gamma activity and ERPs in a visual discrimination task. Clinical Neurophysiology 2002; 113:1742-1753.

29. Van Der Stelt O, Kok A, Smulders F, et al. Cerebral event-related potentials associated with selective attention to color: Developmental 
changes from childhood to adulthood.. Psychophysiology 1998; 35:227-239.

30. Czigler I, Csibra G, Ambro A. Age and information processing: event-related potential studies. European Psychologist 1997; 2:247257.

31. Treisman A, Sato S. Conjunction search revisited. Journal of Experimental Psychology and Human Perceptual Performance 1990; 16:459-478.

32. Eimer $\mathrm{M}$. The N2pc component as an indicator of attentional selectivity. Electroencephalography and Clinical Neurophysiology 1996; 99:225-234.

33. Schubo A, Schröger E, Meinecke C. Texture segmentation and visual search for pop-out targets: An ERP study. Brain Research: Cognitive Brain Research 2004; 21:317-334.

34. Coles M, Smid H, Scheffers M, et al. Mental chronometry and the study of human information processing. In: Rugg $\mathrm{M}$ and Coles $\mathrm{M}$, Eds. Electrophysiology of the Mind. New York: Oxford University Press 1995; 94-95.

35. Sutton S, Braren M, Zublin J, et al. Evoked potential correlates of stimulus uncertainty. Science 1965; 150:1187-1188.

36. Squires NK, Squires KC, Hillyard SA. Two varieties of long-latency positive waves evoked by unpredicatable autditory stimuli in man. Electroencephalography and Clinical Neurophysiology 1975; 39:387401.

37. Knight RT, Scabini D, Woods DL, et al. Contributions of temporalparietal junction to the human auditory P3. Brain Research 1989; 502:109-116.

38. Katayama J, Polich J. Stimulus context determines P3a and P3b. Psychophysiology 1998; 35:23-33.

39. Courchesne E, Hillyard SA, Galambos R. Stimulus novelty, task relevance and the visual evoked potential in man. Electroencephalography and Clinical Neurophysiology 1975; 39:131143.

40. Simmons RF, Graham FK, Miles MA, et al. On the relationship of the P3a and the Novelty P3. Biological Psychology 2001; 56:207-218.

41. He B, Lian J, Spencer KM, et al. A cortical potential imaging analysis of the P300 and novelty P3 components. Human Brain Mapping 2001; 12:120-130.

42. Dien J, Spencer KM, Donchin E. Localization of the event-related potential novelty response as defined by principal components analysis. Cognitive Brain Research 2003; 17:637-650.

43. Buckingham R, Beh HC. The effect of stimulus intensity on the P300. In: Proceedings of the Fifth Australian Psychophysiology Conference; 1995; Wollongong, Australia.

44. Duncan-Johnson CC, Donchin E. On quantifying surprise: The variation in event-related potentials with subjective probability. Psychophysiology 1977; 14:456-467.

45. Duncan-Johnson C, Donchin E. The P300 component of the eventrelated brain potential as an index of information processing. Biological Psychology 1983; 14:1-52.

46. Croft RJ, Gonsalvez CJ, Gabriel C, et al. Target-to-target interval versus probability effects on $\mathrm{P} 300$ in one- and two-tone tasks. Pscyhophysiology 2003; 40:322-328.

47. Nakajima Y, Miyamoto K, Kikuchi M. Estimation of neural generators of cognitive potential P300 by dipole tracing method. No To Shinkei 1994; 46:1059-1065.

48. Tarkka IM, Stokic DS, Baslie LF, et al. Electric source localization of the auditory P300 agrees with magnetic source localization. Electroencephalography and Clinical Neurophysiology 1995; 96:538545 .

49. Knight RT. Neural mechanisms of event-related potentials from lesion studies. In: Rohrbaugh JW, Parasuraman R, and Johnson R Jr, Eds. Event Related Brain Potentials. New York: Oxford University Press, 1990:3-18.

50. Yamaguchi S, Knight RT. Anterior and posterior association cortex contributions to the somatosensory P300. Journal of Neuroscience 1991; 11:2039-2054.

51. Smith ME, Halgren E, Sokolik M, et al. The intracranial topography of the P3 event-related potential elicited during auditory oddball. Electroencephalography and Clinical Neurophysiology 1990; 76:235248.
52. Brookhuis KA, Mulder G, Mulder LJ, et al. The P3 complex as an index of information processing: the effects of response probability. Biological Psychology 1983; 17:277-296.

53. Duncan-Johnson C, Kopell B. The Stroop effect: Brain potentials localize the source of interference. Science 1981; 214:938-940.

54. Ilan AB, Polich J. P300 and response time from a manual Stroop task. Clinical Neurophysiology 1999; 110:367-373.

55. Gonsalvez CJ, Gordon E, Anderson J, et al. Numbers of preceding nontargets differentially affect responses to targets in normal volunteers and patients with schizophrenia: A study of event related potentials. Psychiatry Research 1995; 58:69-75.

56. Gonsalvez CJ, Polich J. P300 amplitude is determined by target-totarget interval. Psychophysiology 2002; 39:388-396.

57. Donchin E, Coles MGH. Is the P300 component a manifestation of context updating? Behavioral and Brain Sciences 1988; 11:357-374.

58. Mccarthy G, Donchin E. A metric for thought: a comparison of P300 latency and reaction time. Science 1981; 211:77-80.

59. Oliver-Rodriguez JC, Guan Z, Johnston VS. Gender differences in late positive components evoked by human faces. Psychophysiology 1999; 36:176-185.

60. Verleger R. Event-related potentials and cognition: A critique of the context updating hypothesis and an alternative interpretation of $\mathrm{P} 3$. Behavioral and Brain Sciences 1988; 11:343-427.

61. Desmedt JE. P300 in serial tasks: An essential post-decision closure mechanism. Progress in Brain Research 1980; 54:682-686.

62. Herbert AM, Gordon GE, Mcculloch DL. A "passive" event-related potential? International Journal of Psychophysiology 1998; 28:11-21.

63. Zenker F, Barajas JJ. Age-Related variations in P300 elicited by active, passive and single-tone paradigms in children. In: IERASG XIV Biennial Symposium; 1996; Lyon, France.

64. Ravden D, Polich J. Habituation of P300 from visual stimuli. International Journal of Psychophysiology 1998; 30:359-365.

65. Geisler MW, Polich J. P300 habituation from visual stimuli? Physiology and Behavior 1994; 56:511-516.

66. Tarkka IM, Stokic DS. Source localization of P300 from oddball, single stimulus, and omitted-stimulus paradigms. Brain Topography 1998; 11:141-151.

67. Polich J, Hoffman D. P300 and handedness. Psychophysiology 1988; 35:497-507.

68. Hopper KD, Patel S, Cann TS, et al. The relationship of age, gender, handedness, and sidedness to the size of the corpus callosum. Academic Radiology 1994; 1:243-248.

69. Driesen NR, Raz N. The influence of sex, age, and handedness on corpus callosum morphology: A meta-analysis. Psychobiology 1995; 23:240-247.

70. Bashore TR. Age-related changes in mental processing revealed by analyses of event related potentials. In: Rohrbaugh JW, Parasuraman R, and Johnson R Jr, Eds. Event Related Brain Potentials. New York: Oxford University Press, 1990:242-278.

71. Smulders FT, Kenemans JL, Schmidt WF, et al. Effects of task complexity in young and old adults: reaction time and P300 latency are not always dissociated. Psychophysiology, 1999; 36:118-125.

72. O'Donnell BF, Friedman S, Swearer JM, et al. Active and passive P3 latency and psychometric performance: influence of age and individual differences. International Journal of Psychophysiology 1992; 12:187-195.

73. Kok A. Age-related changes in involuntary and voluntary attention as reflected in components of the event-related potential (ERP). Biological Psychology 2000; 54(1-3):107-143.

74. Nelson CA, Thomas KM, De Haan M, et al. Delayed recognition memory in infants and adults as revealed by event-related potentials. International Journal of Psychophysiology 1998; 29:145165.

75. Mullis RJ, Holcomb PJ, Diner BC, et al. The effects of aging on the P3 component of the visual event-related potential. Electroencephalography and Clinical Neurophysiology 1985; 62:141149.

76. Knight RT. Decreased response to novel stimuli after prefrontal lesions in man. Electroencephalography Clinical Neurophysiology 1984; 59:9-20 
77. Daffner KR, Mesulam MM, Scinto LF, et al. The central role of the prefrontal cortex in directing attention to novel events. Brain 2000; 123(Pt5):927-939.

78. Jackson SR, Jackson GM, Roberts M. The selection and suppression of action: ERP correlates of executive control in humans. Neuroreport 1999; 10:861-865.

79. Bekker EM, Kenemans JL, Verbaten MN. Source analysis of the N2 in a cued Go/NoGo task. Brain Research Cognitive Brain Research 2005; 22(2):221-231.

80. Barabasz A, Barabasz M, Jensen S, et al. Cortical event-related potentials show the structure of hypnotic suggestions is crucial. The International Journal of Clinical and Experimental Hypnosis 1999; 47:5-22.

81. Polich J, Kok A. Cognitive and biological determinants of P300: an integrative review. Biological Psychology 1995 41(2):103-46.

82. Kosmidis MH, Duncan CC, Mirsky AF. Sex differences in seasonal variations in P300. Biological Psychology 1998; 49:249-268.

83. Nakamura $Y$, Nishimoto $K$, Akamatu M, et al. The effect of jogging on P300 event related potentials. Electromyography and Clinical Neurophysiology 1999; 39:71-74.

84. Hoffman LD, Polich J. EEG, ERPs and food consumption. Biological Psychology 1998; 48:139-11.

85. Roth WT and Cannon EH. Some features of the auditory evoked response in schizophrenics. Archives of General Psychiatry 1972; 27:466-471.

86. Jon Y-W, Polich J. Meta-analysis of P300 and schizophrenia: Patients, paradigms, and practical implications. Psychophysiology 2003; 40:684-701.

87. Guillem F, Bicu M, Pampoulova T, et al. The cognitive and anatomofunctional basis of reality distortion in schizophrenia: A view from memory event-related potentials. Psychiatry Research 2003; 117:137158.

88. Thomas M, Sherwin RS, Murphy J, et al. Importance of cerebral blood flow to the recognition of and physiological responses to hypoglycemia. Diabetes 1997; 46:829-833.

89. Nissenson AR. Epoetin and cognitive function. American Journal of Kidney Diseases 1992; 20:21-24.

90. Begleiter H, Porjesz B, Bihari B, et al. Event-related potentials in boys at risk for alcoholism. Science 1984; 225:483-486.

91. Van Der Stelt O, Geesken R, Gunning WB, et al. P3 scalp topography to target and novel visual stimuli in children of alcoholics. Alcohol 1998; 15:119-36.

92. Porjesz B And Begleiter H. Human brain electrophysiology and alcoholism. In: Tarter R and Thiel DV, Eds. Alcohol and the Brain. New York: Plenum Press, 1985:139-182.

93. Hada M, Porjesz B, Chorlian DB, et al. Auditory P3a deficits in male subjects at high risk for alcoholism. Biological Psychiatry 2001; 49:726-738.

94. Begleiter H, Porjesz B, Reich T, et al. Quantitative trait loci analysis of human event-related brain potentials: P3 voltage. Electroencephalography and Clinical Neurophysiology 1998; 108:244-250.

95. Almasy L, Porjesz B, Blangero J, et al. Genetics of event-related brain potentials in response to a semantic priming paradigm in families with a history of alcoholism. American Journal of Human Genetics 2001; 68:128-135.

96. Tsichiya H, Yamaguchi S, Kobayashi S. Impaired novelty detection and frontal lobe dysfunction in Parkinson's disease. Neuropsychologia 2000; 38:645-654.

97. Lagopoulos J, Gordon E, Barhamali H, et al. Dysfunctions of automatic (P300a) and controlled (P300b) processing in Parkinson's disease. Neurological Research 1998; 20:5-10.

98. Bokura H, Yamaguchi S, Kobayashi S. Event-related potentials for response inhibition in Parkinson's disease. Neurophyschologia 2005; 43(6):967-965.

99. Munte TF, Ridao-Alonso ME, Preinfalk J, et al. An electrophysiological analysis of altered cognitive functions in Huntington disease. Archives of Neurology 1997; 54:1089-1098.

100. Pokryszko-Dragan A, Slotwinski K, Podemski R. Modality-specific changes in P300 parameters in patients with dementia of the Alzheimer type. Medical Science Monitor 2003; 9: 130-134.
101. Olichney JM, Hillert DG. Clinical applications of cognitive eventrelated potentials in Alzheimer's disease. Physical Medicine and Rehabilitation Clinics of North America 2004; 15:205-233.

102.Alonso-Prieto E, Lvarez-Gonzalez MA, Reyes-Verazain A, et al. Use of event related potentials for the diagnosis and follow up of sub clinical disorders of sustained attention in ischemic cerebrovascular disease. Revista de Neurologica 2002; 34:1017-1020.

103. Näätänen R, Escera C. Mismatch negativity: clinical and other applications. Audiology and Neurootology 2000; 5:105-110.

\section{Figures}

Figure 1. Archetypical waveform stimulus-locked representing event-related components of EEG waveforms. N.B., negative waveforms may be represented by upward deflections in traditional notation, which reverses the voltage axis.

$\begin{array}{ll}\text { - Response to frequent stimulus } & \text { A: Baseline } \\ \text { - - Response to infrequent stimulus } & \text { B: N200 } \\ & \text { C: P300 }\end{array}$

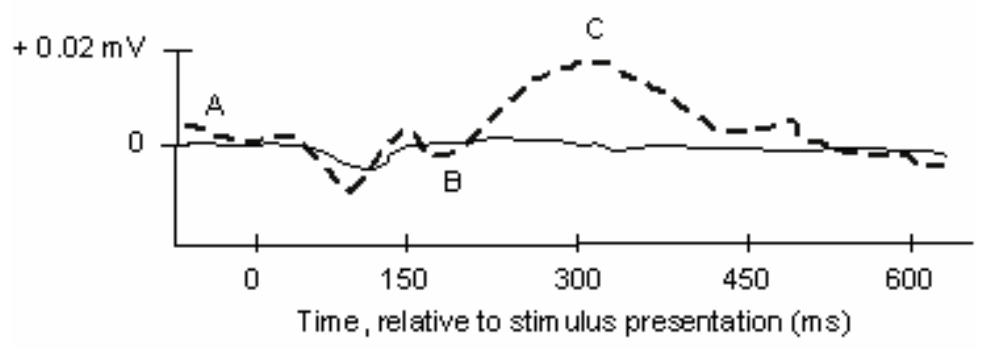

Figure 2. Example multichannel ERP waveforms arranged in a topographic map, from superior "birds-eye" representation $(\mathrm{A}=$ anterior scalp, $\mathrm{P}=$ posterior scalp).

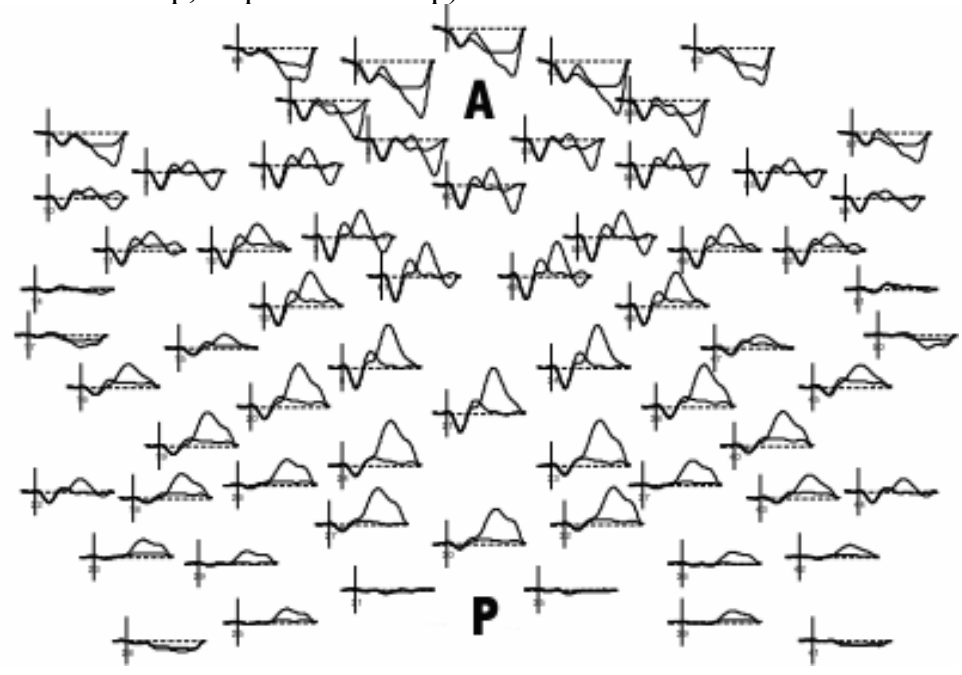

Figure 3. Schematic representation of Oddball (1) and Modified Oddball (2) task paradigms. White box represents a standard or nontarget stimulus; black box represents variant target stimulus; circle represents a deviant, unexpected stimulus presented to the subject.

Time

1
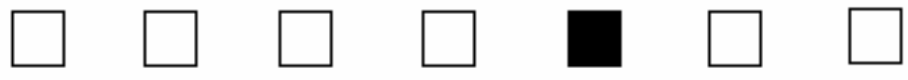

2
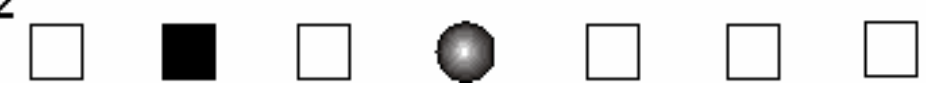